\title{
An approach to a non-contact vital sign monitoring using dual-frequency microwave radars for elderly care
}

\author{
Satoshi Suzuki ${ }^{1}$, Takemi Matsui ${ }^{2}$, Masayuki Kagawa ${ }^{2}$, Takafumi Asao ${ }^{1}, K_{\text {Kentaro Kotani }}{ }^{1}$ \\ ${ }^{1}$ Department of Mechanical Engineering, Faculty of Engineering Science, Kansai University, Osaka, Japan \\ ${ }^{2}$ Department of Management Systems Engineering, Faculty of System Design, Tokyo Metropolitan University, Tokyo, Japan \\ Email: ssuzuki@kansai-u.ac.jp
}

Received 25 April 2013; revised 6 May 2013; accepted 14 May 2013

Copyright (c) 2013 Satoshi Suzuki et al. This is an open access article distributed under the Creative Commons Attribution License, which permits unrestricted use, distribution, and reproduction in any medium, provided the original work is properly cited.

\begin{abstract}
This study aimed to produce a prototype system for non-contact vital sign monitoring of the elderly using microwave radar with the intention of reducing the burdens on monitored individuals and nursing caregivers. In addition, we tested the ability of the proposed prototype system to measure the respiratory and heart rates of the elderly in a nursing home and discussed the systems effectiveness and problems by examining results of real-time monitoring. The prototype system consisted of two $24-\mathrm{GHz}$ microwave radar antennas and an analysis system. The antennas were positioned below a mattress to monitor motion on the body surface for measuring cardiac and respiratory rates from the dorsal side of the subjects $(23.3$ \pm 1.2 years) who would be lying on the mattress. The heart rates determined by the prototype system correlated significantly with those measured by electrocardiography $(r=0.92)$. Similarly, the respiratory rates determined by the prototype correlated with those obtained from respiration curves $(r=0.94)$. Next, we investigated the effectiveness of the prototype system with 7 elderly patients $(93.3 \pm 10.56$ years $)$ at a nursing home. The proposed system appears to be a promising tool for monitoring the vital signs of the elderly in a way that alleviates the need to attach electrodes overnight to confirm patient safety.
\end{abstract}

Keywords: Non-Contact Sensing; Vital Signs; Microwave Radar; Elderly Care

\section{INTRODUCTION}

Recently, the combination of a declining birthrate and an increasing number of elderly persons has become a serious issue in Japan. In particular, Japan is experiencing an aging phenomenon which has not been seen elsewhere in the world. The ratio of those aged 65 and older was already at $20.2 \%$ in 2005 . Japan has the world's fastest aging society [1], and as this increasing tendency continues to rise, officials estimate that elderly people will comprise $23 \%$ of the population by $2010,29 \%$ by 2020 and $40 \%$ by 2050 [1]. The rapidly aging population will create many challenges for Japan in terms of economic planning, healthcare and pensions.

In order to monitor the health of the elderly, devices will be needed that can easily monitor vital signs at home or in hospitals and nursing facilities. Circulatory and respiratory issues in particular pose a risk for the elderly, because they can lead to potentially serious conditions. Therefore, monitoring cardiac and respiratory functions is important. However, on the other hand, long-term monitoring using electrodes places a heavy burden on monitored individuals. Bedsores are common in bedridden elderly persons, and in these cases it is difficult to place electrodes on the skin. For this reason, it is necessary to develop non-contact and unconstrained vital sign measurement methods.

In line with the effects of Japan's aging society, the number of people requiring care is also increasing throughout Japan, and the nation now has an urgent need to cover growing labor shortages in a society that is graying at an alarming rate. In the case of nursing care at home, much of the work of caring for the elderly is undertaken by women and is increasing as the population continues to age. On the other hand, in the case of nursing care at hospitals, serious labor shortages have resulted in new physical and mental burdens on nursing caregivers. Nighttime activity by persons with dementia increases the risk of injury and disrupts caregivers' sleep patterns. Unattended departures from the home also increase risks.

In recent years, various approaches have been proposed to reduce these burdens. Rowe [2] proposed a system called the night monitoring system that was specifi- 
cally designed to alert caregivers when care recipients left their beds and track them as they moved about the house during the night (or other periods when caregivers are asleep). In addition, another study compared the differences in compliance with recommended self-care behavior between users of a self-care and medication compliance device, which was linked to a web-based monitoring system, and those receiving only conventional care [3]. Demiris [4] aimed to investigate elderly behavior using video and image processing while carefully addressing privacy concerns beforehand. Another study proposed an unconstrained and noninvasive vital sign measurement system for measuring heartbeat and respiration to monitor health status at home or in hospitals and nursing facilities [5].

The requirements for the system relating to nursing care are as follows: 1) monitoring of activity and vital signs must not be a burden for the elderly; 2) patient physical condition and changes are accurately and comprehensibly displayed; and 3) in case of a rapid change in physical condition, the system should promptly notify a doctor or nursing caregiver.

In order to cover aforesaid problems, especially 1), various noninvasive sensing techniques have been developed to measure human vital signs. Several attempts of noninvasive pulse monitoring have been made using a strain gauge [6] and pressure sensors [7]. Wang et al. reported a method to measure heart beat and respiration via a subject's back using a polyvinylidene fluoride film piezoelectric polymer sensor [8]. Such non-electrode methods are useful for patients with severe burns. However, they do have inherent limitations in contact method.

We have previously reported a non-contact method that uses a ceiling-attached microwave radar to monitor the respiratory rates of subjects in bed through thick bedding [9] and attached it to the back of a chair to monitor changes of autonomic activation [10]. Microwave radar has the following characteristics: 1) it can detect movement of a remote object without having to make contact; and 2) microwaves can be transmitted through most solid substances except metal or water. The aim of using this radar method was to measure motion on an extremely small scale which occurs on the body surface as a result of cardiac and respiratory activity. This method was originally developed for searching for survivors under earthquake rubble [11,12]. We have also previously developed a non-contact vital sign monitoring system using dual-frequency microwave radars $(10 \mathrm{GHz}$ and $24 \mathrm{GHz}$ ) for measuring the vital signs of casualty patients inside a moving ambulance [13]. This system was able to simultaneously measure the respiratory and heart rates of patients from outside an isolator without touching the patient, regardless of whether the ambulance was in motion or stopped and idling. If it becomes possible to use this prototype system developed for ambulances for overnight monitoring of the elderly, it is advantageous because the elderly person is free from the burden of monitoring equipment and electrodes.

Therefore, this study aimed to develop a prototype system for non-contact vital sign monitoring to confirm the safety of the elderly with the intention of reducing the burden on monitored individuals. Moreover, we tested the ability of the prototype to measure the respiratory and heart rates of the elderly at a nursing home and discuss its effectiveness and limitations while examining the results of in situ monitoring.

\section{SYSTEM DESIGN}

\subsection{Prototype System for Non-Contact Vital Sign Monitoring Prototype System for Non-Contact Vital Sign Monitoring}

The prototype system for non-contact vital sign monitoring consists of two $24-\mathrm{GHz}$ microwave radar antennas to ensure sufficient resolution stability, and an automated analysis system. Each radar has the same specification; average output power of about $7 \mathrm{~mW}$ (max. $10 \mathrm{~mW}$ ), antenna gain of $10 \mathrm{dBi}$ and diffusion angle of approximately $40^{\circ}$. In order to avoid crossing the frequency of each antenna, the frequency of the sensors changes at 24.110 GHz and 24.150 GHz. Both antennas are housed in a small rectangular box $(8 \mathrm{~cm} \times 5 \mathrm{~cm} \times 3 \mathrm{~cm}$ with a $1.1 \mathrm{~cm} \times 1.2 \mathrm{~cm}$ quadra-pole plane antenna) containing an oscillator and a microwave antenna.

The system is controlled by a personal computer with custom sampling and display programs written in LabVIEW (Version 8.5, National Instruments, TX, CO). The analog outputs of the two microwave radars are transferred to a personal computer through an A/D converter and analyzed in real time. The respiratory and heart rates, as well as the cardiac and respiratory curves from both the non-contact (microwave radar) and contact (electrocardiogram (ECG) and respiration sensor) instruments are displayed on a graphical terminal in real time (Figure 1).

\subsection{Analytical Method}

The output signals from both $24 \mathrm{GHz}$ microwave radars and reference outputs were sampled by the A/D converter with a sampling frequency $100 \mathrm{~Hz}$. In order to reduce noise and split the output signals from each radar, the respiratory and heart rate signals were each routed through a band-pass filter. For cardiac monitoring, the band-pass filter was set between $0.5 \mathrm{~Hz}$ and $2.5 \mathrm{~Hz}$. For respiratory monitoring, the filter was set between 0.05 $\mathrm{Hz}$ and $0.5 \mathrm{~Hz}$. These band-pass filters cover ranges from 30 to 150 heart beats per minute and from 3 to 30 respi- 


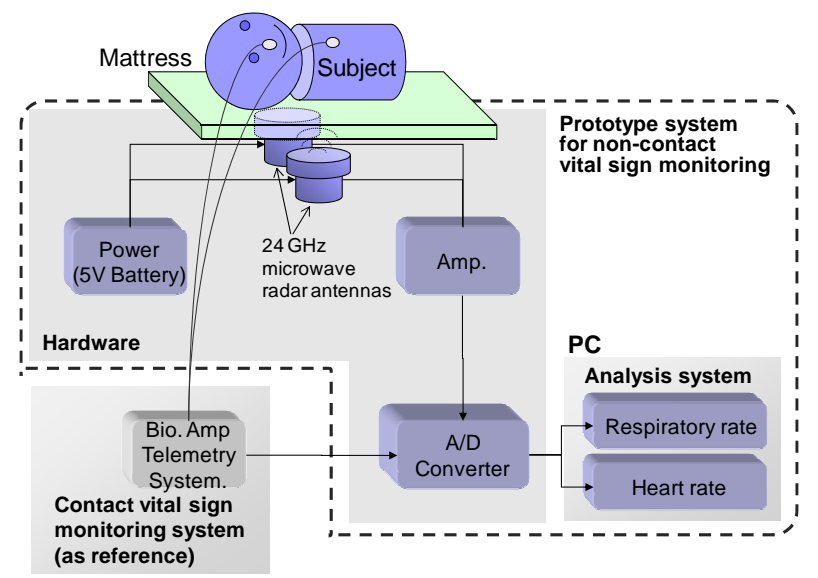

Figure 1. Schematic diagram of the experimental setup to test a non-contact vital sign monitoring system that uses double microwave radar antennas placed under a mattress.

rations per minute. Each signal for respiratory and heart rate was derived by the band-pass filters. The band pass filter for the $24 \mathrm{GHz}$ microwave radar for cardiac-monitoring was set between 0.5 and $2.5 \mathrm{~Hz}$. For the $10 \mathrm{GHz}$ microwave radar for respiratory monitoring, the filter was set between 0.05 and $0.5 \mathrm{~Hz}$. These band pass filters cover ranges 30 - 150 heart beats per minute and 3 - 30 respirations per minute for the respiration, respectively. After band-pass filtering, each signal was processed by a Fast Fourier Transform (FFT) using a Hanning window.

It was assumed that the output signal is biased to one side of each of the two antennas according to body posture and positioning. Therefore, in order to stably calculate heart rate, an analytical process was conducted with the following steps: 1) the result from the FFT conducted for each signal from the respective microwave radar after band-pass filtering set at between $0.5 \mathrm{~Hz}$ and $2.5 \mathrm{~Hz}$ was multiplied; 2) the frequency of the peak signal between $0.5 \mathrm{~Hz}$ and $2.5 \mathrm{~Hz}$ was assumed to correspond to heart rate. The same analytical process was conducted between $0.05 \mathrm{~Hz}$ and $0.5 \mathrm{~Hz}$ to calculate respiratory rate.

The sequence of the analytical process was achieved by an automated system written in LabVIEW (Figure 2). The correlation coefficient between the heart rate acquired by the non-contact vital sign monitoring system and that by the contact monitoring system was examined. Statistical analysis was performed using StatMate III Software (ATMS Co. Ltd., Tokyo, Japan).

\section{EXPERIMENTS}

\subsection{Experiment 1: Laboratory Investigation with Adults}

Initially, we investigated the proposed system and assumed some conditions of body posture and positioning. The experiments were conducted with 8 healthy males (age: $23.3 \pm 1.2$ years; range: 21 - 25 years).
The mattress (Preglar-mattress, KE-551, Paramount Bed Co., Tokyo, Japan; $191 \mathrm{~cm} \times 91 \mathrm{~cm} \times 8 \mathrm{~cm}$ size) used in the experiments was made from polyester and was chosen because it is the same type as that used widely in nursing homes for the elderly in Japan. The 24 $\mathrm{GHz}$ microwave radar antennas were positioned below the mattress. The radar monitored the microwave reflection modulated by cardiac motions from the dorsal side of the subject. In order to measure the motion of the body surface near the heart, microwave radar antennas were positioned around the shoulder blades. The radars were placed $70 \mathrm{~cm}$ above the top of the mattress according to measurements in a database of Japanese body size (Japanese Body Dimension Data, 1997-1998). The sensors were set up in positions divided evenly in the lateral direction (Figure 3). Each subject lay quietly on their back on the mattress while measurements were conducted.

Under real-life conditions, it is likely the patient will change body posture and positioning in the bed. Therefore, we performed experiments under two conditions: 1) considering particular body posture (face-up, lateral and

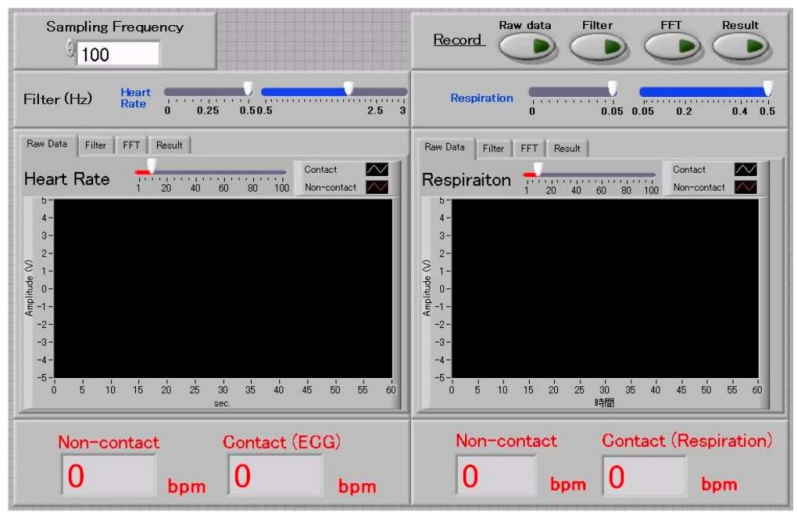

Figure 2. Graphic display of automated sampling and indicating program written in LabVIEW. A Fast Fourier Transform of the microwave radar analogue outputs was conducted with a Hanning window to derive the respiratory and heart rates.

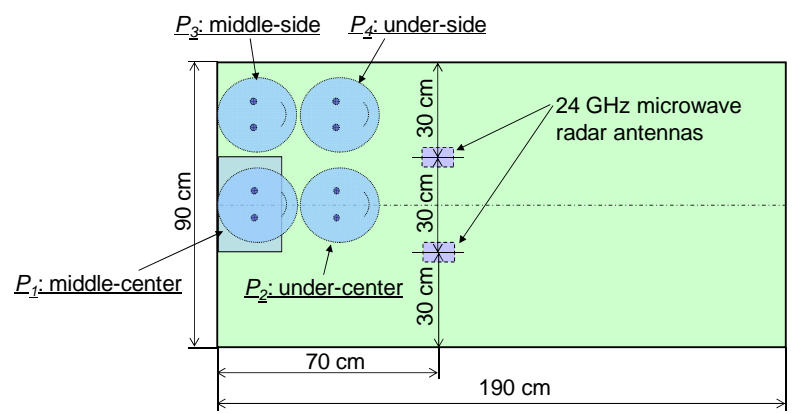

Figure 3. Mattress size, installation position of double microwave radar antennas of prototype system for non-contact vital sign monitoring and body positions based on the head position at middle-center (P1), under-center (P2), middleside (P3) and under-side (P4) in the face-up position. 
face-down positions at middle-center (P1) at the head position); and 2) considering body positioning (condition of head position at middle-center (P1), under-center (P2), middle-side (P3) and under-side (P4) in the face-up position) (Figure 3).

The measurement with the prototype system for noncontact vital sign monitoring using microwave radar was performed for $5 \mathrm{~min}$. At the same time, for reference, the subject's precordial ECG was measured with electrodes, and the respiratory curve was measured using a thermistor respiration sensor (TR511G, Nihon Koden Co., Tokyo, Japan) inserted into the nasal cavity. Both contact-monitoring systems were connected to a multi-channel telemetry system (WEB-5500, Nihon Koden Co., Figure 1).

The study protocol was reviewed and approved by the Committee on Human Research of the Faculty of System Design, Tokyo Metropolitan University.

\subsection{Experiment 2: Trial Operation with Elderly at a Nursing Home}

To verify the effectiveness of the prototype system in its intended setting, we investigated its capability with 7 elderly volunteers (age: $93.3 \pm 10.56$ years; range: 80 to 104 years) at a nursing home.

Table 1 shows the physical characteristics of the volunteers. We requested that only patients deemed fit enough by a medical doctor to participate in the study were included. We obtained the agreement for this survey directly from the volunteers or from their families when it was difficult to determine whether or not the patient consented as some patients have cognitive dysfunction.

We used the same prototype system and analytical sys-

Table 1. Individual data on the physical characteristics of the 7 elderly volunteers ${ }^{\mathrm{a}}$.

\begin{tabular}{|c|c|c|c|}
\hline Subject & Age & Disease state & Condition \\
\hline$S e_{1}$ & 104 & $\begin{array}{l}\text { Cerebral infarction, } \\
\text { High-blood pressure }\end{array}$ & Require nursing care \\
\hline $\mathrm{Se}_{2}$ & 98 & $\begin{array}{c}\text { Cerebral infarction, } \\
\text { Dementia }\end{array}$ & $\begin{array}{l}\text { Feed through a } \\
\text { gastromy tube }\end{array}$ \\
\hline $\mathrm{Se}_{3}$ & 100 & $\begin{array}{l}\text { Cerebral infarction, } \\
\text { High-blood pressure }\end{array}$ & Require nursing care \\
\hline $\mathrm{Se}_{4}$ & 104 & Cardiac dysrhythmia & Require nursing care \\
\hline $\mathrm{Se}_{5}$ & 85 & $\begin{array}{l}\text { Chronic constipation, } \\
\text { Bone fracture }\end{array}$ & Require nursing care \\
\hline $\mathrm{Se}_{6}$ & 82 & $\begin{array}{l}\text { Alzheimer's dementia, } \\
\text { Cerebral infarction }\end{array}$ & Require nursing care \\
\hline $\mathrm{Se}_{7}$ & 80 & $\begin{array}{l}\text { Cerebral infarction, } \\
\text { Dementia }\end{array}$ & $\begin{array}{l}\text { Feed through a } \\
\text { gastromy tube }\end{array}$ \\
\hline Average & 93.29 & & \\
\hline$S D$ & 10.56 & & \\
\hline
\end{tabular}

${ }^{\text {a }}$ Mean age was $93.3 \pm 10.56$ years (range 80 - 104 years). SD: standard deviation. tem of the previous experiment. The radar antennas were set up in the same position. Before the investigation, we acquired pulse waves of each volunteer using a cuff with an infrared diode (OLV-3100, Nihonkoden Co.) on the middle finger in place of ECG. This was performed because a medical doctor suggested that electrodes should not be pasted directory onto the skin for an extended period as it was considered a burden for elderly patients. We monitored the pulse wave for about $1 \mathrm{~min}$ and checked the correlation between the heart rate estimated from data acquired by the prototype system and the heart rate calculated by pulse wave before each investigation. The investigation was conducted from 9 pm to 7 am after verifying that the correlation between heart rates calculated by both systems was $>0.9$.

\section{RESULTS}

\subsection{Result 1: Investigation with Young Subjects}

Figures 4(a) and (b) shows the sample signal from the prototype system with a period of 1 min. Figure 4(a) shows the signals corresponding to the cardiac oscillation measured by the prototype system and ECG. Figure 4(b) shows the signals corresponding to the respiration measured by the prototype system and thermistor sensor of Subject A. From these figures, it can be confirmed that the prototype system output exhibited cyclic oscillations with human cardiac motion which correspond to the R-R intervals of the ECG. Additionally, a sample of cyclic oscillation with a period of 1 min was observed and corresponded well with the respiratory curves measured by the thermistor respiration sensor.

The heart and respiratory rates determined by the microwave radars using our custom program with the FFT were compared with those measured by the contact methods. Table 2(a) shows the results for each posture for both contact and non-contact measurement methods. Table 2(b) shows the results for each position using both contact and non-contact measurement methods.

For each posture and position, heart rate as determined by the prototype system of non-contact vital sign monitoring using the $24 \mathrm{GHz}$ microwave radar correlated significantly and stably with the rates measured by ECG with electrodes (posture: mean $r=0.92, p<0.01$; position: mean $r=0.94, p<0.01$ ). The respiratory rates measured by the same prototype system also correlated significantly with the respiratory rates measured by thermistor sensor (posture: mean $r=0.94, p<0.01$; position: mean $r=0.94, p<0.01)$.

\subsection{Result 2: Trial Operation with Elderly Patients in Nursing Home}

Figure 5 shows results of change in heart rate (Figure $\mathbf{5 ( a ) )}$ and respiratory rate (Figure $\mathbf{5 ( b ) )}$ ) at $2 \mathrm{~h}$ intervals 
Table 2. Results of investigation with young subjects

a) Results of correlations of respiratory and heart rates between the contact and non-contact measurement methods in each posture in all 7 volunteers.

\begin{tabular}{cccc}
\hline Position & & Correlation & $p$ \\
\hline \multirow{2}{*}{ Dorsal } & Respiratory rate & 0.92 & ${ }^{* *}$ \\
& Heart rate & 0.90 & ${ }^{* *}$ \\
\multirow{2}{*}{ Prone } & Respiratory rate & 0.97 & ${ }^{* *}$ \\
& Heart rate & 0.95 & ${ }^{* *}$ \\
\multirow{2}{*}{ Decubitus } & Respiratory rate & 0.93 & ${ }^{* *}$ \\
& Heart rate & 0.90 & ${ }^{* *}$ \\
\hline
\end{tabular}

${ }^{* *} p<0.01$.

b) Results of correlations of respiratory and heart rates between the contact and non-contact measurement methods in each position in all 7 volunteers.

\begin{tabular}{cccc}
\hline Pillow position & & Correlation & $p$ \\
\hline \multirow{2}{*}{ middle-center } & Respiratory rate & 0.93 & ${ }^{* *}$ \\
& Heart rate & 0.95 & ${ }^{* *}$ \\
under-center & Respiratory rate & 0.96 & ${ }^{* *}$ \\
& Heart rate & 0.97 & ${ }^{* *}$ \\
middle-center & Respiratory rate & 0.93 & ${ }^{* *}$ \\
& Heart rate & 0.92 & ${ }^{* *}$ \\
under-center & Respiratory rate & 0.95 & ${ }^{* *}$ \\
H* $^{*}<0.01$. & Heart rate & 0.90 & ${ }^{* *}$ \\
\cline { 2 - 3 } & & &
\end{tabular}

during the night as monitored by the prototype system. In fact, the system confirmed noise deriving from body motion as an artifact. For general change of heart and respiratory rate monitored at $2 \mathrm{~h}$ intervals during the night, there was a slight difference between each subject. However, it was confirmed that the heart rate and respiratory rate decreased as the night progressed and that these changes became more rapid as dawn approached.

Figure 6 shows the result of the respiratory pattern of Volunteer 1 acquired from the prototype system. The oscillation halted for about $20 \mathrm{~s}$ after continuing short-period oscillations, and cheyne-stokes breathing was confirmed. Thus, it seems that this volunteer exhibited symptoms of sleep apnea.

\section{DISCUSSION}

It is assumed that the relationship between sleeping posture and positioning of the radar antennas influences the accuracy of estimation of heart and respiratory rates.

These can also be measured with an electromagnetic field simulator, a usable method under specific circumstances, in order to verify the results. However, when there are changes in what is being measured, like posture change, and when there are changes in the measurement

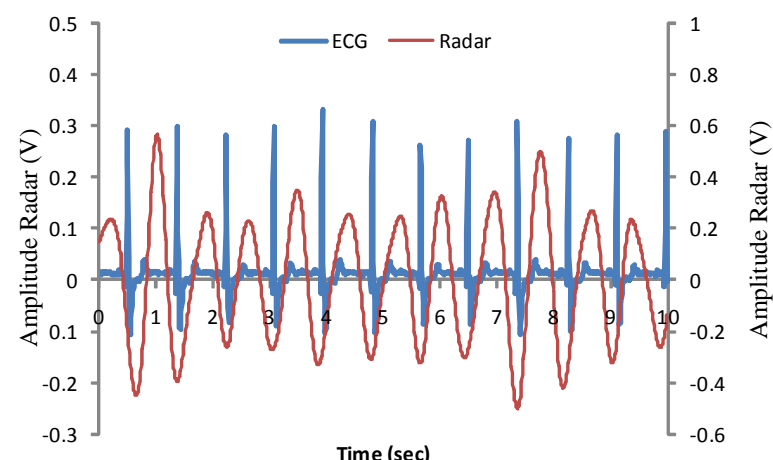

(a)

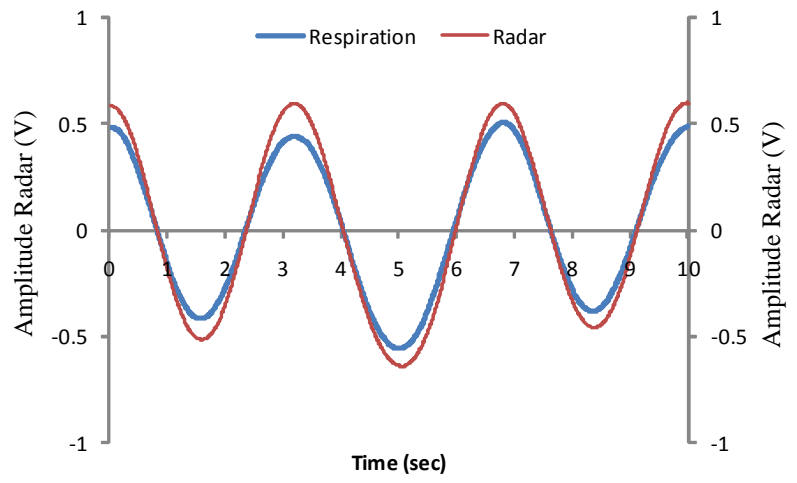

(b)

Figure 4. Samples of output signals from the $24 \mathrm{GHz}$ microwave radar output for heart beat and respiration. (a) Cyclic oscillations with human cardiac motion corresponding to the $\mathrm{R}-\mathrm{R}$ intervals of the electrocardiography; and (b) cyclic oscillations with the respiratory motions of human chest wall corresponding to the respiratory curves measured by a contact thermistor sensor.

parameters like a change of positioning, it is impossible to exactly verify the readings. Therefore, in this study, we verified the effectiveness of our proposed system through laboratory experiments on whether the results depend on sleeping posture and antenna positioning.

The heart and respiratory rates determined by the prototype system had a generally high correlation with the rates measured by ECG using electrodes independent of posture. Moreover, both the heart and respiratory rates in the prone position had a slightly higher correlation than those of other postures. A possible reason for this difference is that the motion of the abdomen is large and that there is little or no motion on the dorsal side induced by respiration. Additionally, the heart is located slightly offcenter; therefore, the motion on the body surface on the foreside is larger than that on the dorsal side. Whatever the reason, the heart and respiratory rates determined by the prototype system had a high correlation independent of posture. Therefore, it was assumed that this system would be effective.

The heart and respiratory rates determined by the prototype system had a generally high correlation with the 


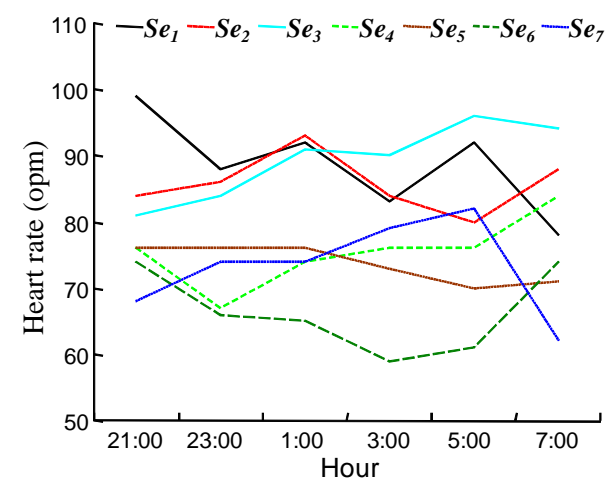

(a)

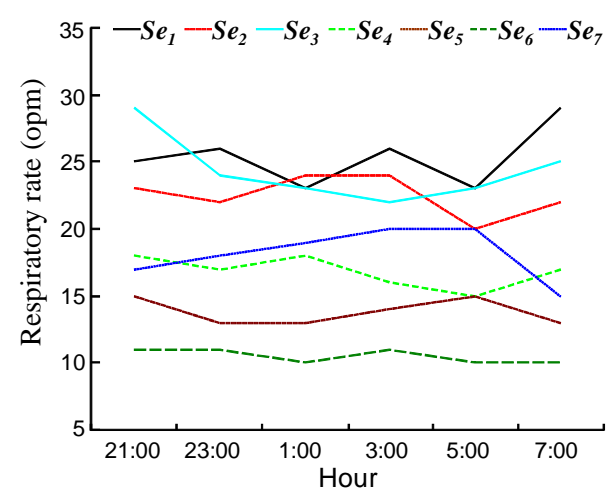

(b)

Figure 5. (a) Results of change of heart; and (b) respiratory rates at $2 \mathrm{~h}$ intervals during the night as monitored by the prototype system. It was confirmed that the heart and the respiratory rates decrease as the night progressed and were increased further with the approach of dawn.

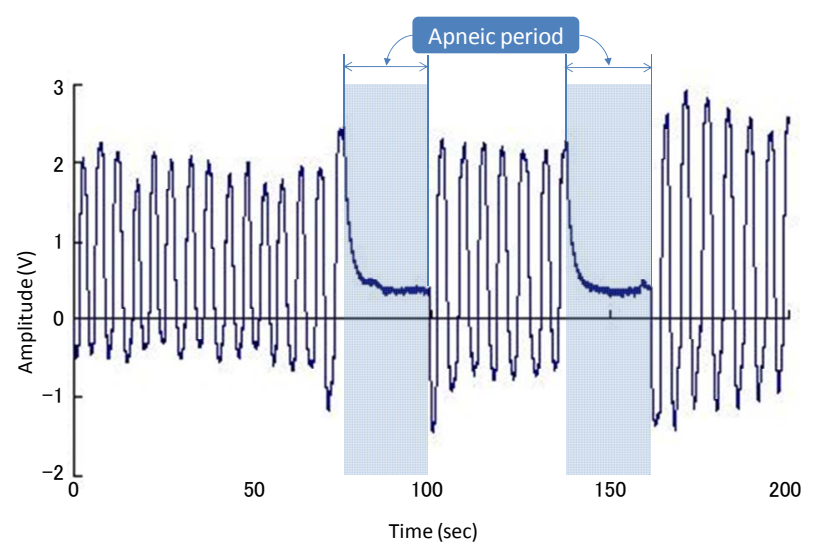

Figure 6. A sample result of the respiratory pattern of volunteer 1 acquired from the prototype system. The oscillation halted for about $20 \mathrm{~s}$ after a continuing short period of oscillations and cheyne-stokes breathing was confirmed.

rates measured by ECG in each head positions, that is, in each measurement position on the dorsal side. The correlation was slightly less in the head position of the middle-side and under-side, namely, the sleeping posture misaligned the measurement position and position of the radar antennas. It is considered, therefore, that the relation between sleeping posture and positioning of the radar antennas influences the accuracy of estimation of heart and respiratory rates which depend on the diffusion angle of the antenna. Thus, it is necessary to consider both the installation position and diffusion angle of the antenna in real use.

A certain level of cardiac output from elderly patients in healthy condition is maintained at rest. However, myocardial distensibility decreases and myocardial relaxation time is often extended by indurated tissue around the heart with age. Consequently, late filling of left ventricular early distention occurs. In response, compensatory atrial contraction occurs. Although, if the compensatory contraction is reduced by atrial fibrillation and tachyarrhythmia, it can lead to heart failure. Cardiac arrest by diastolic failure, as a result, is seen with an increased frequency in elderly patients [14]. Therefore, it is necessary to investigate whether the prototype system and our proposed method can be used to capture such an occurrence and predict cardiac failure.

On the other hand, it is well known that the respiratory function, such as ventilatory and heart rate response to hypoxia and hypercapnia, slow down with age [15]. There are some characteristics of physiological changes related to age including a reduction in arterial oxygen saturation [16], partial pressure of oxygen [17] and the presence of the neuronal isoform of nitric oxide synthase in skeletal muscle [18]. Despite decreased tidal volume, the minute ventilation volume of the elderly is the same as that of adults, and as a result, the respiratory rate of the elderly tends to decrease. Thus, respiration of the elderly is rapid and shallow [19]. On the other hand, during sleep, the prevalence of sleep apnea tends to increase with age. The prevalence of any type of sleep apnea (central and obstructive) is increased monotonically with age [20]. Apneas tended to occur predominantly in stage 1 - 2 of sleep and seemed to be an exaggeration of the periodicity that is typical of this state. In addition, the elderly with apnea remain in this stage of sleep throughout the night. There is a report where apnea of $>10 \mathrm{~s}$ was confirmed in 38\% of elderly subjects [21]. The characteristics of respiration during sleep in the elderly can be summarized as follows: 1) respiration pattern is rapid and shallow; and 2) increased apnea during sleep. These two characteristics are apparent in the results of volunteer 1 in the present study.

\section{CONCLUSIONS}

As a conclusion of this study, we found that our prototype system could effectively monitor heart and respiratory rate of subjects; the dual $24 \mathrm{GHz}$ microwave radar antenna outputs exhibited cyclic oscillations that corresponded to cardiac motion and respiratory motion of the 
chest wall. Additionally, we investigated the effectiveness of the prototype system in relation to the elderly at a nursing home.

We also developed a module for the prototype system to send images of patient analysis via the internet to a medical doctor who stays at home over night. According to the doctor, it was easy to verify the safety of the monitored elderly patients by viewing their heart and respiratory rates. Therefore, it is assumed that this prototype system and the concept of this monitoring method are effective. The proposed system appears promising for future monitoring of vital signs of elderly patients while relieving the heavy burden of electrodes placed overnight for detection of adverse events. Furthermore, this prototype system will contribute to reducing burdens on elderly and nursing caregivers.

\section{REFERENCES}

[1] Ministry of Internal Affairs and Communications (2012) Statistical topics No. 14. http://www.stat.go.jp/data/topics/topi140.htm

[2] Rowe, M.A., Kelly, A., Horne, C., Lane, S., Campbell, J., Lehman, B., Phipps, C., Keller, M. and Benito A.P. (2009) Reducing dangerous nighttime events in persons with dementia by using a nighttime monitoring system. Alzheimer's \& Dementia, 5, 419-426. doi:10.1016/j.jalz.2008.08.005

[3] Artinian, N.T., Harden, J.K., Kronenberg, M.W., Vander Wal, J.S., Daher, E., Stephens, Q. and Bazzi, R.I. (2003) Pilot study of a Web-based compliance monitoring device for patients with congestive heart failure. Heart Lung, 32, 226-233. doi:10.1016/S0147-9563(03)00026-8

[4] Demiris, G., Oliver, D.P., Giger, J., Skubic, M. and Rantz, M. (2009) Older adults' privacy considerations for vision based recognition methods of eldercare applications. Technology and Health Care, 17, 41-48.

[5] Tanaka, S., Matsumoto, Y. and Wakimoto, K. (2002) Unconstrained and non-invasive measurement of heart-beat and respiration periods using a phonocardiographic sensor. Medical \& Biological Engineering \& Computing, 40, 246-252. doi:10.1007/BF02348132

[6] Ciaccio, E.J., Hiatt, M., Hegyi, T. and Drzewiecki, G.M. (2007) Measurement and monitoring of electrocardiogram belt tension in premature infants for assessment of respiratory function. BioMedical Engineering OnLine, 6, $1-11$.

[7] Jacobs, J., Embree, P., Glei, M., Christensen, S. and Sullivan, P. (2004) Characterization of a novel heart and respiratory rate sensor. In: Hudson, D.L., Liang, Z.P. and Dumont, G., Eds., Proceedings of the 26th Annual International Conference of the IEEE Engineering in Medicine and Biology Society, IEEE Service Center, San Francisco, 2223-2226.

[8] Wang, F., Tanaka, M. and Chonan, S. (2006) Development of a wearable mental stress evaluation system using PVDF film sensor. Journal of Advanced Science, 18,

\section{0-173. doi:10.2978/jsas.18.170}

[9] Uenoyama, M., Matsui, T., Yamada, K., Suzuki, S., Takase, B., Suzuki, S. and Ishihara, M. (2006) Non-contact respiratory monitoring system using a ceiling-attached microwave antenna. Medical \& Biological Engineering \& Computing, 44, 835-840. doi:10.1007/s11517-006-0091-8

[10] Suzuki, S., Matsui, T., Imuta, H., Uenoyama, M., Yura, H. and Ishihara, M. (2008) A novel autonomic activation measurement method for stress monitoring: Non-contact measurement of heart rate variability using a compact microwave radar. Medical \& Biological Engineering \& Computing, 46, 709-714. doi:10.1007/s11517-007-0298-3

[11] Chen, K.M., Huang, Y. and Zhang, J. (2000) Microwave life-detection systems for searching human subjects under earthquake rubble or behind barrier. IEEE Transactions on Biomedical Engineering, 27, 105-113. doi:10.1109/10.817625

[12] Chen, K.M., Misra, D., Wang, H., Chuang, H.R. and Postow, E. (1986) An X-band microwave life-detection system. IEEE Transactions on Biomedical Engineering, 33 697-702. doi:10.1109/TBME.1986.325760

[13] Suzuki, S., Matsui, T., Kawahara, H., Ichiki, H., Shimizu, J., Kondo, Y., Gotoh, S., Yura, H., Takase, B. and Ishihara, M. (2009) A non-contact vital sign monitoring system for ambulances using dual-frequency microwave radars. Medical \& Biological Engineering \& Computing, 47, 101-105. doi:10.1007/s11517-008-0408-x

[14] Gottdiener, J.S., McClelland, R.L., Marshall, R., Shemanski, L., Furberg, C.D., Kitzman, D.W., Cushman, M., Polak, J., Gardin, J.M., Gersh, B.J., Aurigemma, G.P. and Manolio, T.A. (2002) Outcome of congestive heart failure in elderly persons: Influence of left ventricular systolic function. The cardiovascular health study. Annals of Internal Medicine, 137, 631-639. doi:10.7326/0003-4819-137-8-200210150-00006

[15] Richard, S.K. and Charles, W.D. (1973) Attenuation of the ventilatory and heart rate responses to hypoxia and hypercapnia with aging in normal men. Journal of Clinical Investigation, 52, 1812-1819. doi:10.1172/JCI107363

[16] Cerveri, I., Zoia, M.C., Fanfulla, F., Spagnolatti, L., Berrayah, L., Grassi, M. and Tinelli, C. (1995) Reference values of arterial oxygen tension in the middle-aged and elderly. American Journal of Respiratory and Critical Care Medicine, 152, 934-941. doi:10.1164/ajrccm.152.3.7663806

[17] Gunnarsson, L., Tokics, L., Brismar, B. and Hedenstierna, G. (1996) Influence of age on circulation and arterial blood gases in man. Acta Anaesthesiologica Scandinavica, 40, 237-243. doi:10.1111/j.1399-6576.1996.tb04426.x

[18] Krumpe, P.E., Knudson, R.J., Parsons, G. and Reiser, K. (1985) The aging respiratory system. Clinics in Geriatric Medicine, 1, 143-175.

[19] Krieger, B.P., Isber, J., Breitenbucher, A., Throop, G. and Ershowsky, P. (1997) Serial measurements of the rapidshallow-breathing index as a predictor of weaning outcome in elderly medical patients. Chest, 112, 1029-1034. doi:10.1378/chest.112.4.1029

[20] Bixler, E.O., Vgontzas, A.N., Ten Have, T., Tyson, K. 
and Kales, A. (1998) Effects of age on sleep apnea in men: I. Prevalence and severity. American Journal of Respiratory and Critical Care Medicine, 157, 144-148. doi:10.1164/ajrccm.157.1.9706079

[21] Shore, E.T., Millman, R.P., Silage, D.A., Chung, D.C. and Pack, A.I. (1985) Ventilatory and arousal patterns during sleep in normal young and elderly subjects. Journal of Applied Physiology, 59, 1607-1615. 